\title{
Thymoquinone Attenuates Myocardial Ischemia/Reperfusion Injury Through Activation of SIRT1 Signaling
}

\author{
Yunyang Lu ${ }^{a}$ Yingda Fenga,b Dan Liuc ${ }^{\mathrm{a}}$ Zhiran Zhang ${ }^{c} \quad$ Kai Gao $^{d}$ \\ Wei Zhang ${ }^{d}$ Haifeng Tang ${ }^{\text {a }}$
}

Institute of Materia Medica, School of Pharmacy, Fourth Military Medical University, Xi'an, ${ }^{\mathrm{b}}$ Department of Ultrasound, Xijing Hospital, Fourth Military Medical University, Xi'an, 'Department of Pharmacy, 210 Hospital of PLA, Dalian, 'Department of Pharmacy, Xijing Hospital, Fourth Military Medical University, Xi'an, China

\section{Key Words}

Thymoquinone - Myocardial ischemia/reperfusion injury • SIRT1 • Ac-p53 • Mitochondrial oxidative stress $\cdot$ Apoptosis

\begin{abstract}
Background/Aims: Myocardial ischemia/reperfusion (MI/R) injury is a leading factor responsible for damage in myocardial infarction, resulting in additional injury to cardiac tissues involved in oxidative stress, inflammation, and apoptosis. Thymoquinone (TQ), the main constituent of Nigella sativa L. seeds, has been reported to possess various biological activities. However, few reports regarding myocardial protection are available at present. Therefore, this study was conducted aiming to investigate the protective effect of TQ against MI/R injury and to clarify its potential mechanism. Methods: MI/R injury models of isolated rat hearts and neonatal rat cardiomyocytes were established. The Langendorff isolated perfused heart system, triphenyltetrazolium chloride staining, gene transfection, TransLaser scanning confocal microscopy, and western blotting were employed to evaluate the cardioprotection effect of TQ against MI/R injury. Results: Compared with the MI/R group, TQ treatment could remarkably improve left ventricular function, decrease myocardial infarct size and production of lactate dehydrogenase (LDH), and attenuate mitochondrial oxidative damage by elevating superoxide dismutase (SOD) activity and reducing production of hydrogen peroxide $\left(\mathrm{H}_{2} \mathrm{O}_{2}\right)$ and malonaldehyde (MDA). Moreover, the cardioprotective effect of TQ was accompanied by up-regulated expression of SIRT1 and inhibition of p53 acetylation. Additionally, TQ treatment could also enhance mitochondrial function and reduce the number of apoptotic cardiomyocytes. Nonetheless, the cardioprotective effect of TQ could be mitigated by SIRT1 inhibitor sirtinol and SIRT1 siRNA, respectively, which was achieved through inhibition of the SIRT1 signaling pathway. Conclusions: The findings in this study demonstrate that TQ is efficient in attenuating MI/R injury through activation of the SIRT1 signaling pathway, which can thus reduce mitochondrial oxidative stress damage and cardiomyocyte apoptosis.
\end{abstract}

Y. Lu and Y. Feng contributed equally to this work. 


\section{Cellular Physiology Cell Physiol Biochem 2018;47:1193-1206

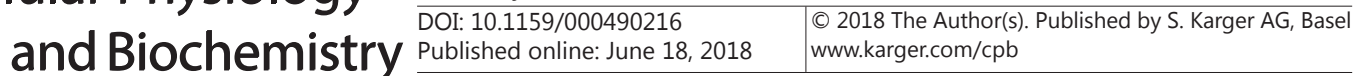

Lu et al.: Thymoquinone Attenuates Myocardial Ischemia/Reperfusion Injury

\section{Introduction}

Coronary heart disease (CHD) is the leading cause of death and disability worldwide, and myocardial ischemia/reperfusion (MI/R) is one of the major manifestations of CHD [1, 2]. The underlying mechanism of I/R injury is complicated and unclear, and may be related to factors such as mitochondrial dysfunction, overproduction of reactive oxygen species (ROS), reduction of nitric oxide bioavailability, and intracellular calcium overload [3, 4]. Recently, a study has shown that shock wave therapy may serve as an effective option for ischemic heart disease [5]. Nevertheless, no effective therapy for preventing reperfusion injury is available to date [6]; thus, further study is urgently needed.

Thymoquinone (TQ) is isolated from Nigella sativa $L$. seeds, which are used as herbal medicine and spice in some African, Asian, and European countries [7-9]. Notably, TQ has been reported to possess a wide range of biological activity including antitumor [10, 11], anti-inflammation [12, 13], anti-oxidation [14], and immunoregulatory effects [15]. Furthermore, the protective effect of TQ on MI/R injury has been demonstrated in numerous organs, including the liver [16], spinal cord [17], brain [18], kidney [19] and testis [20]. Additionally, Gonca and Kurt [21] and Ojha et al [14] have demonstrated that TQ could protect cardiomyocytes from MI/R injury. However, the underlying molecular mechanism of TQ remains unclear.

TQ is a pharmacologically active quinone derivative, which is also a naturally derived compound featured by a conjugated double bond system responsible for its reactivity and the intracellular process known as "redox cycling" [22]. TQ-dependent redox cycling can lower the cellular reductive balance by reducing the (NADH/NAD ${ }^{+}$ratio; meanwhile, it may sensitize the oxidative metabolic pathway by increasing substrate flux to mitochondria [23].

Silent mating-type information regulator 2 homolog 1 (SIRT 1 ) is an NAD -dependent histone deacetylase, which plays a critical role in many cellular pathways, such as aging, metabolism, stress, and apoptosis [24, 25]. Furthermore, elevated intracellular NAD+ level activate the SIRT1-dependent metabolic pathways [26]. In addition, SIRT1 was recently found to play an important regulatory role in cardiovascular disease through activation of FOXO to down-regulate the apoptotic molecules and to reduce oxidative stress for cardioprotection [27]. Of them, p53 is a transcription factor modulated by SIRT 1 , which can profoundly affect cell survival and death. Acetylated p53 can promote oxidative stress-induced apoptosis, whereas SIRT1 can render deacetylation of the acetylated p53, thus altering the cell apoptosis process [28].

Based on the structural characteristics of TQ and molecular function of SIRT1, it is speculated that TQ may be related to SIRT1. In this study, we aimed to investigate the protective effect of TQ against MI/R injury and to clarify whether it was related to the SIRT1 signaling pathway.

\section{Materials and Methods}

\section{Animals}

All animal experiments were approved by the Institute of Animal Care and Use Committee of the Fourth Military Medical University, which were conducted in strict accordance with the Guide for the Care and Use of Laboratory Animals (NIH publication No. 85-23, National Institutes of Health Publication, Washington, DC, USA, revised 1996). One-day-old Sprague-Dawley (SD) rats and 8-week-old male SD rats (250-300 g) were supplied by the Laboratory Animal Center of the Fourth Military Medical University. All rats were maintained on a regular $12 \mathrm{~h}$ light/12 h dark cycle at a constant temperature of $22 \pm 1^{\circ} \mathrm{C}$ and humidity of 40-70\%; standard food and sterile water could be accessed ad libitum.

Main reagents and kits

TQ was provided by Bailingwei Scientific (Beijing, China; purity $\geq 98 \%$ ). Dimethyl sulfoxide (DMSO), $4^{\prime}, 6^{\prime}$-diamino-2'-phenylindole (DAPI), trypsin, and collagenase type II were all purchased from Sigma- 


\section{Cellular Physiology Cell Physiol Biochem 2018;47:1193-1206

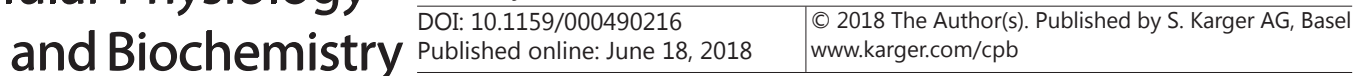

Lu et al.: Thymoquinone Attenuates Myocardial Ischemia/Reperfusion Injury

Aldrich (St. Louis, MO). Dulbecco's modified Eagle's medium/nutrient mixture F-12 (DMEM/F-12) and fetal bovine serum (FBS) were obtained from Gibco Laboratories (Life Technologies, Inc., Burlington, ON, Canada). Cell Counting Kit-8 (CCK-8) was obtained from 7 Sea Biotechnology Co., Ltd. (Shanghai, China). Terminal deoxynucleotidyl transferase dUTP nick end labeling (TUNEL) kits were bought from Roche Molecular Biochemicals (Mannheim, Germany). Triphenyltetrazolium chloride (TTC) was supplied by Sigma-Aldrich. In addition, kits for measuring lactate dehydrogenase (LDH), super oxygen dehydrogenases (SOD), hydrogen peroxide $\left(\mathrm{H}_{2} \mathrm{O}_{2}\right)$, methane dicarboxylic aldehyde (MDA), and glutathione/oxidized glutathione (GSH/ GSSG) were purchased from Jiancheng Bioengineering Institute (Nanjing, China). Mitochondrial extraction kits were obtained from Solarbio Science and Technology Co., Ltd. (Beijing, China). Antibodies against SIRT1, acetylated-p53 (ac-p53), B-cell lymphoma-2 (Bcl2), Bcl-2-associated X protein (Bax), succinate dehydrogenase (SDH, complex II), and cytochrome coxidase (COX, complex IV) were obtained from Cell Signaling Technology, Inc., (Beverly, MA). Sirtinol, SIRT1 siRNA, antibodies against cleaved caspase-3 and $\beta$-actin were purchased from Santa Cruz Biotechnology, Inc., (Santa Cruz, CA). Additionally, goat anti-rabbit and goat anti-mouse secondary antibodies were purchased from the Zhongshan Biotechnology Co., Ltd. (Beijing, China). Notably, all other chemicals and reagents used in this study were of analytical grade and purchased from approved organizations.

\section{MI/R model by Langendorff-perfused system ex vivo}

SD rats were anaesthetized with intraperitoneal injection of $10 \%$ chloral hydrate supplemented with $0.1 \mathrm{~mL}$ heparin (5000 IU/mL) prior to the experiment. As described previously [29], hearts were quickly isolated and arrested in ice-cold perfusate, which were then transferred to the Langendorff apparatus (Radnoti LLC., Monrovia, CA) by inserting a perfusion cannula into the aorta under a constant pressure of 80 $\mathrm{mmHg}$. Subsequently, hearts were perfused with Krebs-Henseleit (K-H) solution (containing $120 \mathrm{mM} \mathrm{NaCl}$, $4.7 \mathrm{mM} \mathrm{KCl}, 2.5 \mathrm{mM} \mathrm{CaCl}_{2}, 1.2 \mathrm{mM} \mathrm{MgSO}_{4}, 1.2 \mathrm{mM} \mathrm{KH}_{2} \mathrm{PO}_{4}, 25 \mathrm{mM} \mathrm{NaHCO}_{3}, 11 \mathrm{mM}$ glucose, $2 \mathrm{mM}$ pyruvate acid, and $0.5 \mathrm{mM}$ EDTA) equilibrated with a gas mixture comprising $95 \% \mathrm{O}_{2}$ and $5 \% \mathrm{CO}_{2}$ at $37{ }^{\circ} \mathrm{C}$ to produce a PH of 7.4. Later, the isolated hearts were subjected to $45 \mathrm{~min}$ of global no-flow ischemia, followed by 60 min of reperfusion to induce MI/R. Meanwhile, the left ventricular developed pressure (LVDP) and the rate of change of pressure development $(+\mathrm{dP} / \mathrm{dt}$ max) were monitored using a transducer (Model 100BP, Biopac System Inc., Goleta, CA).

\section{Preparation of primary neonatal rat cardiomyocyte cultures}

Neonatal rat cardiomyocytes were isolated as previously described [30]. Briefly, hearts were isolated and transferred to cold phosphate-buffered saline (PBS). The ventricles were then minced into small pieces, followed by digestion by trypsin ( $4 \mathrm{mg} / \mathrm{mL})$ and collagenase type II $(1 \mathrm{mg} / \mathrm{mL})$ at $37^{\circ} \mathrm{C}$. Next, cardiomyocytes were recovered by centrifugation before they were resuspended in growth medium supplemented with 90\% DMEM/F-12 1:1 medium, 10\% FBS, $100 \mathrm{U} / \mathrm{mL}$ of penicillin and streptomycin. The cardiomyocytes were then incubated at $37{ }^{\circ} \mathrm{C}$ and $5 \% \mathrm{CO}_{2}$ in a humidified incubator. After $90 \mathrm{~min}$, the supernatant was collected and cardiomyocytes were then re-plated onto either 96-well plates at a density of $1 \times 10^{4}$ cells/ well or into tissue culture flasks or petri dishes at a concentration of $5 \times 10^{4}$ cells/dish. After $48 \mathrm{~h}$, the neonatal rat cardiomyocytes were washed and cultured in serum-free maintenance medium (DMEM/F-12 $1: 1$ medium) at $37^{\circ} \mathrm{C}$ and $5 \% \mathrm{CO}_{2}$ for the duration of the whole experiment.

\section{Preparation of simulated IR model of primary neonatal cardiomyocytes in vitro}

The primary neonatal rat cardiomyocytes were maintained in anoxic medium containing similar concentrations of potassium, hydrogen, and lactate as those in vivo. As described previously [31], the cardiomyocytes were exposed to an ischemic buffer ( $\mathrm{pH}$ 6.5) containing $137.0 \mathrm{mM} \mathrm{NaCl}, 12.0 \mathrm{mM} \mathrm{KCl}, 0.49$ $\mathrm{mM} \mathrm{MgCl}, 0.9 \mathrm{mM} \mathrm{CaCl}{ }_{2} \cdot 2 \mathrm{H}_{2} \mathrm{O}, 4.0 \mathrm{mM}$ HEPES, $10.0 \mathrm{mM}$ deoxyglucose, $0.75 \mathrm{mM}$ sodium dithionate, and $20.0 \mathrm{mM}$ lactate. Subsequently, the cardiomyocytes were incubated in a humidified cell culture incubator at $37{ }^{\circ} \mathrm{C}$ in the presence of $5 \% \mathrm{CO}_{2}$ and $21 \% \mathrm{O}_{2}$ for $2 \mathrm{~h}$. Later, reperfusion was initiated by transferring the cardiomyocytes into the normal DMEM for $4 \mathrm{~h}$.

\section{Experimental protocol}

Step 1 was designed to evaluate the effects of TQ on the normal isolated rat hearts. Briefly, TQ was completely dissolved in DMSO before diluted using K-H buffer to different concentrations. Specifically, the 


\section{Cellular Physiology Cell Physiol Biochem 2018;47:1193-1206

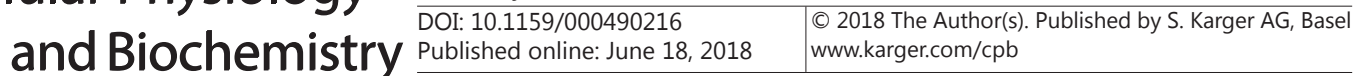

Lu et al.: Thymoquinone Attenuates Myocardial Ischemia/Reperfusion Injury

hearts were stochastically divided into four experimental groups, namely, control, TQ 2.5, TQ 5, and TQ $10 \mu \mathrm{mol} / \mathrm{L}$ groups. Afterwards, the hearts were treated with TQ for $5 \mathrm{~min}$ after $10 \mathrm{~min}$ of equilibration, followed by perfusion with K-H buffer for 105 min. The control group was perfused with K-H buffer for 110 $\mathrm{min}$, and the dose and schedule were selected with reference to previous experiments and studies [32]. The hemodynamic results demonstrated that $1 \mu \mathrm{mol} / \mathrm{L}$ TQ had almost no effect on improving LVDP during reperfusion compared with the MI/R group ( $>0.05$ ), whereas the effect of $15 \mu \mathrm{mol} / \mathrm{L}$ TQ on improving LVDP during reperfusion was not superior to that of $10 \mu \mathrm{mol} / \mathrm{L}$ TQ compared with that of the TQ $10 \mu \mathrm{mol} /$ $\mathrm{L}+\mathrm{MI} / \mathrm{R}$ group $(\mathrm{P}>0.05)$. As a result, the TQ concentrations of $2.5,5$, and $10 \mu \mathrm{mol} / \mathrm{L} \mathrm{TQ}$ were adopted in this study.

Step 2 was designed to examine the role of SIRT1 in the myocardial protection after TQ pretreatment in the isolated rat hearts. Firstly, the rat hearts were randomly divided into five experimental groups, namely, control, MI/R, TQ $2.5 \mu \mathrm{mol} / \mathrm{L}+\mathrm{MI} / \mathrm{R}$, TQ $5 \mu \mathrm{mol} / \mathrm{L}+\mathrm{MI} / \mathrm{R}$ and TQ $10 \mu \mathrm{mol} / \mathrm{L}+\mathrm{MI} / \mathrm{R}$ groups. The isolated hearts were subjected to $45 \mathrm{~min}$ of global no-flow ischemia after $10 \mathrm{~min}$ of equilibration, followed by $60 \mathrm{~min}$ of reperfusion to induce MI/R. Later, the hearts were treated with TQ for 5 min prior to the MI/R experiment. Secondly, the hearts were further randomly divided into four experimental groups, namely, MI/R, sirtinol + $\mathrm{MI} / \mathrm{R}, \mathrm{TQ}+\mathrm{MI} / \mathrm{R}$ o, and TQ + sirtinol + MI/R groups. After 10 min of equilibration, the isolated hearts were treated with TQ or an admixture of $3.75 \mu \mathrm{mol} / \mathrm{L}$ sirtinol for $5 \mathrm{~min}$ prior to the MI/R experiment, and the dose and schedule were determined with reference to previous experiments and studies [31].

Step 3 was designed to examine the role of SIRT1 in the myocardial protection after TQ pretreatment in SIR injured cardiomyocytes. Firstly, the cardiomyocytes were randomly divided into control and TQ (1, $2,4,8,16$, and $32 \mu \mathrm{mol} / \mathrm{L}$ ) groups. Cell viability was then detected based on CCK-8 assay. Secondly, the cardiomyocytes were further randomly divided into five groups, namely, control, SIR, TQ $1 \mu \mathrm{mol} / \mathrm{L}+\mathrm{SIR}$, TQ $2 \mu \mathrm{mol} / \mathrm{L}+\mathrm{SIR}$ and TQ $4 \mu \mathrm{mol} / \mathrm{L}+\mathrm{SIR}$ groups. Subsequently, cells were treated with TQ at the concentrations of 1,2 , and $4 \mu \mathrm{mol} / \mathrm{L} 24 \mathrm{~h}$ prior to the IR experiment. Thirdly, the cardiomyocytes were randomly divided into four groups, namely, SIR, SIRT1 siRNA + SIR, TQ + SIR, and TQ + SIRT1 siRNA + SIR groups and were then transfected with scramble siRNA or SIRT1 siRNA (100 pM) using the LipoFiter liposomal transfection reagent (Hanbio Biotechnology, Shanghai, China) in strict accordance with the manufacturer's instructions. Later, cells were incubated in antibiotics-free medium for $6 \mathrm{~h}$ and washed in warmed medium, followed by at least $24 \mathrm{~h}$ of equilibration to ensure expression before usage. The transfection efficiency of siRNA was confirmed by western blotting. Specifically, cells were treated with $4 \mu \mathrm{mol} / \mathrm{L}$ TQ $24 \mathrm{~h}$ prior to the SIR experiment.

\section{TTC staining for detecting myocardial infarct size}

Upon the completion of the MI/R experiment, the hearts were removed at the end of reperfusion and incubated in $1 \%$ TTC solution at $37^{\circ} \mathrm{C}$ for $30 \mathrm{~min}$ in the dark, followed by immersion in $4 \%$ paraformaldehyde solution for $24 \mathrm{~h}$. Subsequently, 6 cross-sectional slices were taken and imaged under a digital camera. The percentage of infarct area (white area) of the total ventricular area was calculated using Image J software.

\section{Myocardial mitochondrial and cytosol isolation}

Myocardial mitochondria were isolated using a mitochondrial extraction kit in strict accordance with the kit instructions. In brief, the tissues or cells were mixed with ice cold lysis buffer; subsequently, the mixture was ground into homogenate and transferred into a centrifuge tube. The homogenate was then subjected to $5 \mathrm{~min}$ of centrifugation at $1000 \mathrm{~g}$ twice. The supernatant was collected, followed by another 10 min of centrifugation at $12000 \mathrm{~g}$. The supernatant was again collected, containing most of the cytoplasmic protein; the sediments were mostly mitochondria. The sediments were washed with $0.5 \mathrm{~mL}$ washing buffer and centrifuged at $1000 \mathrm{~g}$ for $5 \mathrm{~min}$ to collect the supernatant for another $10 \mathrm{~min}$ of centrifugation at 12000 $g$. All the centrifugation processes were executed at $4{ }^{\circ} \mathrm{C}$. Finally, the sediments made up of highly purified mitochondria were collected.

\section{Mitochondrial oxidative damage assessment}

SOD activity, MDA, and $\mathrm{H}_{2} \mathrm{O}_{2}$ levels, and GSH/GSSG ratio in the mitochondria were assessed to determine the oxidative damage using the commercially available kits in strict accordance with the manufacturer's instructions. In addition, expression levels of SDH and COX were quantified using western blotting.

\section{KARGER}




\section{Cellular Physiology Cell Physiol Biochem 2018;47:1193-1206 and Biochemistry Published online: June 18, $2018 \quad \begin{aligned} & \text { DOI: 10.1159/000490216 } 2018 \text { The Author(s). Published by S. Karger AG, Basel } \\ & \text { www.karger.com/cpb }\end{aligned}$}

Lu et al.: Thymoquinone Attenuates Myocardial Ischemia/Reperfusion Injury

\section{Cell viability determination}

The primary neonatal rat cardiomyocytes were treated with TQ at concentrations of $1 \mu \mathrm{mol} / \mathrm{L}, 2$ $\mu \mathrm{mol} / \mathrm{L}$ and $4 \mu \mathrm{mol} / \mathrm{L} 24 \mathrm{~h}$ prior to the SIR experiment, and 6 parallel replicates were prepared. Specifically, $10 \mu \mathrm{L}$ CCK-8 reagent was added into each well in the dark in strict accordance with the kit instructions. Afterwards, the 96-well plates were incubated in a humidified cell culture incubator in the presence of 5\% $\mathrm{CO}_{2}$ and $21 \% \mathrm{O}_{2}$ at $37^{\circ} \mathrm{C}$ for $2 \mathrm{~h}$. Optical density, which indicated cell viability, was later analyzed at $450 \mathrm{~nm}$ using a microplate reader (SpectraMax 190, Molecular Devices, Inc., CA). Images were taken using a 600D camera (Canon Company, Tokyo, Japan).

\section{Cardiomyocyte apoptosis and myocardial damage}

Apoptosis was assessed using TUNEL assay in strict accordance with the manufacturer's instructions. As described previously [33], cells were washed twice with PBS after TQ treatment, followed by fixing in $4 \%$ paraformaldehyde for $30 \mathrm{~min}$. Subsequently, cells were incubated in $0.1 \%$ Triton X-100 for 15 min on ice, washed with PBS, and covered with $80 \mu \mathrm{L}$ TUNEL reaction mixture. Next, all cell samples were incubated in a humidified chamber for $1 \mathrm{~h}$ at $37^{\circ} \mathrm{C}$ in the dark. Then, cells were washed with PBS three times and stained with DAPI $(10 \mu \mathrm{g} / \mathrm{mL})$. Finally, apoptotic cells were visually identified in 10 selected fields and photographed at high-power magnification $(\times 200)$ with an Olympus FV1000 (Olympus, Tokyo, Japan) confocal microscope.

LDH release, a commonly used marker for cardiomyocyte damage, was assessed in K-H buffer and culture medium using the commercially available kits in strict accordance with the kit instructions, so as to determine the cardiomyocyte damage.

\section{Western blotting}

Western blotting was performed as previously described [34]. Briefly, the treated hearts and cells were collected and washed with ice-cold PBS prior to the addition of lysis buffer (containing $20 \mathrm{mM}$ Tris- $\mathrm{HCl}$ at the $\mathrm{pH}$ of 7.5; $150 \mathrm{mM} \mathrm{NaCl}$; $1 \mathrm{mM}$ EDTA; $1 \mathrm{mM} \mathrm{EGTA;} \mathrm{1 \%} \mathrm{Triton;} 2.5 \mathrm{mM}$ sodium pyrophosphate; $1 \mathrm{mM}$ $\beta$-glycerophosphate; $1 \mathrm{mM} \mathrm{Na}_{3} \mathrm{VO}_{4}$; and $1 \mu \mathrm{g} / \mathrm{mL}$ leupeptin). Heart tissue of SD rats was cut into pieces before addition of lysis buffer. Afterwards, protein concentration was quantified using the bicinchoninic acid assay protein assay kit. Then, protein was separated into equivalent amounts by $8 \%-10 \%$ SDS-PAGE and transferred onto the polyvinylidene fluoride membranes (PVDF) membranes. Later, the PVDF membranes were blocked with Tween-20 Tris-buffered saline (containing $10 \mathrm{mM}$ Tris- $\mathrm{HCl}$ at the $\mathrm{pH}$ of $7.5 ; 100 \mathrm{mM}$ $\mathrm{NaCl}$; and $20 \mathrm{mM}$ of $0.1 \%$ Tween) supplemented with $5 \%$ fat free milk, and incubated overnight at $4{ }^{\circ} \mathrm{C}$ with antibodies against SIRT1, ac-p53, Bcl2, Bax, Cleaved Caspase3, SDH, COX (1:1000), and $\beta$-actin (1:3000). Subsequently, the PVDF membranes were incubated with secondary antibodies for $2 \mathrm{~h}$ at room temperature. Finally, images were scanned by using a Bio-Rad imaging system (Bio-Rad Laboratories Inc., Hercules, CA) and densities were determined using Image Lab software (Bio-Rad Laboratories Inc.).

\section{Statistical analysis}

All values are presented as the mean \pm standard deviation (SD). Differences between experimental groups were examined using one-way analysis of variance, followed by Tukey's test for post hoc test. All statistical tests were conducted using the Graph Pad Prism software version 5.0. A P-value of less than 0.05 was considered as statistically significant.

\section{Results}

Effects of TQ on cardiac function, infarct size, LDH release, and SIRT1 expression of normal isolated rat hearts

Compared with the control group, TQ treatment at the concentrations of 2.5, 5 and 10 $\mu \mathrm{mol} / \mathrm{L}$ showed no effects on LVDP after $110 \mathrm{~min}$ of perfusion ( $\mathrm{P}>0.05$, Fig. 1B). Moreover, TQ treatment would not induce irreversible damage on LDH release (Fig. 1C) or infarct size (Fig. 1D) relative to the control group ( $\mathrm{P}>0.05)$. However, TQ pretreatment could remarkably up-regulate SIRT1 expression in normal rat hearts in a concentrations-dependent manner (P $<0.5$, Fig. 1E). 
Effects of $T Q$ pretreatment on cardiac function, infarct size and LDH release of $M I / R$ injured isolated rat hearts

The LVDP values had markedly decreased compared with baseline levels after the MI/R procedure $(\mathrm{P}<0.01)$. Compared with the MI/R group, TQ treatment at the concentrations of $2.5,5$ and $10 \mu \mathrm{mol} / \mathrm{L}$ could notably enhance functional recovery of the post-ischemic hearts, as was exhibited by the evidently higher and concentrations-dependent values of LVDP and $+d p / d t$ throughout the reperfusion period ( $<<0.01$, Fig. $2 \mathrm{~A})$. Compared with the control group, the total LDH release from the coronary effluent in MI/R group had markedly increased, while TQ pretreatment apparently lowered LDH release in a concentrationdependent manner (compared with the MI/R group, $\mathrm{P}<0.01$, Fig. 2B). Images of hearts after TTC staining are shown in Fig. 2C, revealing few infarct areas in the control group.

Fig. 1. Effects of $\mathrm{TQ}$ on $\mathrm{LDH}$ release, cardiac function, infarct size, and SIRT1 expression of normal isolated rat hearts. (A) Chemical structure of TQ. (B) Representative diagrams of cardiac function. (C) LDH release. (D) Representative images of hearts. (E) Representative images of SIRT1 protein level detected by western blotting. All results are expressed as the mean $\pm \mathrm{SD}, \mathrm{n}=6$. ${ }^{\text {aa }} \mathrm{P}<0.01$ compared with the control group; ${ }^{\mathrm{b}} \mathrm{P}<0.05$ compared with the TQ 2.5 $\mu \mathrm{mol} / \mathrm{L}$ group; ${ }^{\text {bb }} \mathrm{P}<0.01$ compared with the TQ $2.5 \mu \mathrm{mol} / \mathrm{L}$ group; and ${ }^{c} \mathrm{P}<0.01$ compared with the TQ 5 $\mu \mathrm{mol} / \mathrm{L}$ group. TQ, thymoquinone (Molecular weight: 164; molecular formula: $\mathrm{C}_{10} \mathrm{H}_{12} \mathrm{O}_{2}$ ).

Fig. 2. Effects of TQ pretreatment on LDH release, cardiac function, and infarct size of MI/Rinjured isolated rat hearts. (A) Representative diagrams of cardiac function. (B) LDH release. (C) Representative images of hearts. All results are expressed as the mean $\pm \mathrm{SD}, \mathrm{n}=6$. ${ }^{\text {a }}<0.05$ compared with the control group; ${ }^{a} \mathrm{P}<0.01$ compared with the control group; ${ }^{b b} \mathrm{P}<0.01$ compared with the MI/R group; ${ }^{c c} \mathrm{P}<0.01$ compared with the TQ $2.5 \mu \mathrm{mol} / \mathrm{L}+\mathrm{MI} / \mathrm{R}$ group; and ${ }^{\mathrm{d}} \mathrm{P}<0.05$ compared with the TQ $5 \mu \mathrm{mol} / \mathrm{L}+\mathrm{MI} / \mathrm{R}$ group. MI/R, myocardial ischemia/reperfusion (45 min/60 min).
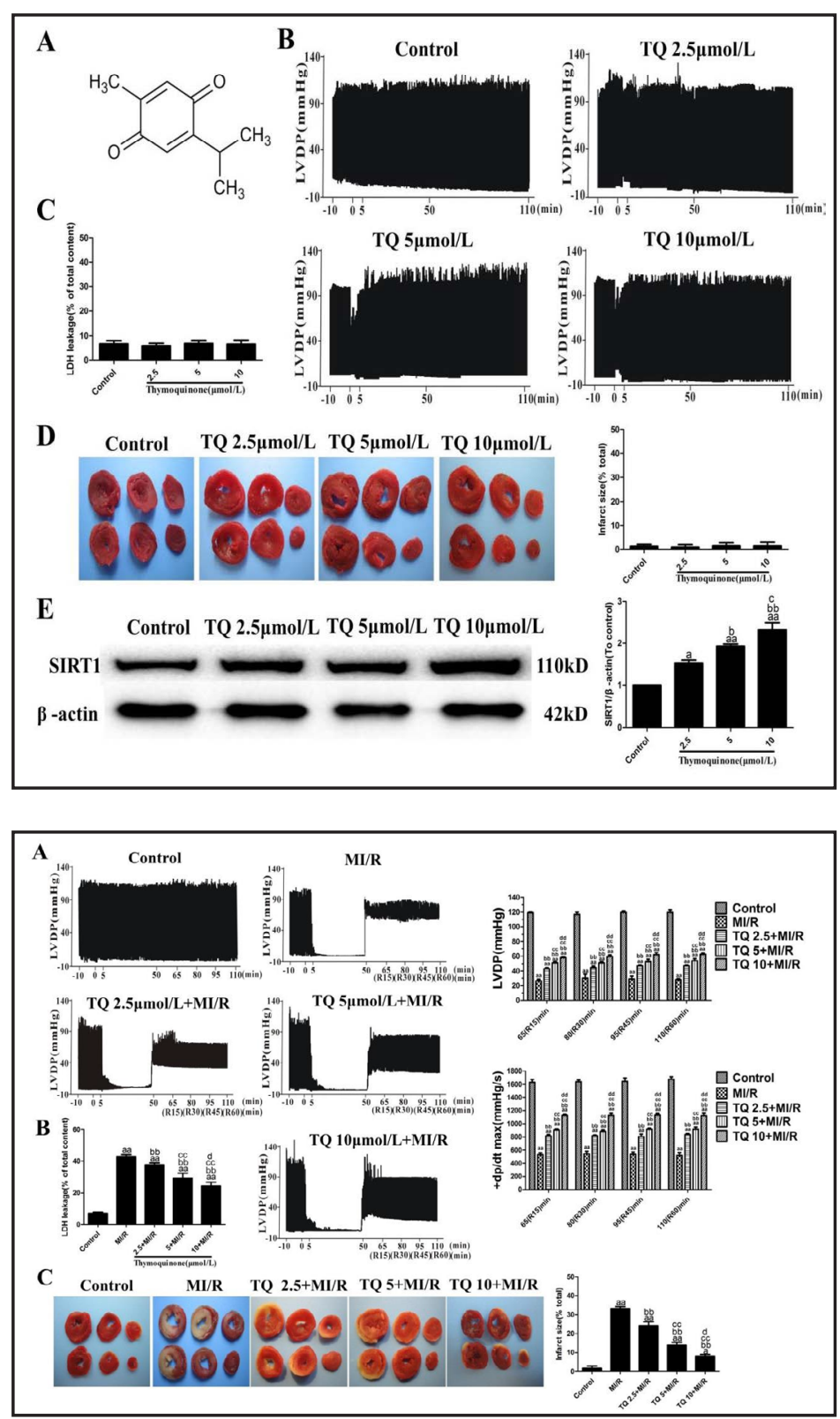
The percentage of the infarct area was $33.25 \pm 1.06 \%$ after MI/R, which had been markedly reduced by TQ treatment to $8.01 \pm 0.96 \%$ (compared with the MI/R group, $\mathrm{p}<0.05$ ), indicating the potential cardioprotective effect of TQ during MI/R process.

\section{Effects of TQ pretreatment on mitochondrial oxidative damage of MI/R-injured isolated rat hearts}

As shown in Fig. 3, MI/R treatment down-regulated the activity of mitochondrial SDH, COX, and SOD (Fig. 3B, P < 0.01), decreased GSH/GSSG ratio (Fig. 3E, $\mathrm{P}<0.01$ ), and upregulated the levels of mitochondrial MDA (Fig. 3D, $\mathrm{P}<0.01$ ) and $\mathrm{H}_{2} \mathrm{O}_{2}$ (Fig. 3C, $\mathrm{P}<0.01$ ). Compared with the MI/R group, TQ treatment significantly up-regulated the expression levels of SDH and COX, elevated mitochondrial SOD activity, and enhanced GSH/GSSG ratio in the TQ + MI/R group (compared with the MI/R group, $\mathrm{P}<0.01$ ). Consistently, levels of mitochondrial MDA and $\mathrm{H}_{2} \mathrm{O}_{2}$ were remarkably lower in the TQ + MI/R group than in MI/R group $(\mathrm{P}<0.01)$.

Effects of TQ pretreatment on expression levels of SIRT1, ac-p53, Bcl2, Bax and Cleaved Caspase 3 of MI/R-injured isolated rat hearts

As observed in Fig. 3, TQ treatment markedly up-regulated SIRT1 expression while down-regulating acetylation of p53 (compared with the MI/R group, $\mathrm{P}<0.01$ ). Moreover, compared with the MI/R group, TQ pretreatment notably increased the $\mathrm{Bcl} 2 / \mathrm{Bax}$ ratio but down-regulated Cleaved Caspase-3 expression in a concentration-dependent manner $(\mathrm{P}<$ $0.05)$.

Effects of TQ and sirtinol pretreatment on cardiac function, infarct size, and LDH release of $M I / R$-injured isolated rat hearts

Compared with the MI/R group, sirtinol + MI/R treatment displayed no significant influence on cardiac function $(\mathrm{P}>0.05)$. However, sirtinol pretreatment notably attenuated the functional recovery of the post-ischemic hearts, as was demonstrated by the markedly lower values of LVDP and $+\mathrm{dp} / \mathrm{dt}$ throughout the reperfusion period (compared with the TQ $+\mathrm{MI} / \mathrm{R}$ group, $\mathrm{P}<0.01$, Fig. 4A). Compared with the MI/R group, the total LDH release from the coronary effluent in the TQ + MI $/$ group had been evidently reduced $(\mathrm{P}<0.01$, Fig. 4B).

Fig. 3. Effects of $\mathrm{TQ}$ pretreatment on SIRT1 and acetylation of p53, apoptosis and oxidative stress injury in MI/R-injured isolated rat hearts. (A) Representative western blotting results are presented. (B) SOD activity. (C) $\mathrm{H}_{2} \mathrm{O}_{2}$ formation. (D) MDA content. (E) GSH/GSSG ratio. All results are expressed as the mean $\pm \mathrm{SD}, \mathrm{n}=6 .{ }^{\mathrm{a}} \mathrm{P}<0.05$ compared with the control group; aa $\mathrm{P}<0.01$ compared with the control group; ${ }^{\text {b }}<0.05$ compared with the $\mathrm{MI} / \mathrm{R}$ group; ${ }^{\mathrm{bb}} \mathrm{P}<0.01$ compared with the $\mathrm{MI} / \mathrm{R}$ group; ${ }^{\mathrm{C}} \mathrm{P}<0.05$ compared with the TQ $2.5 \mu \mathrm{mol} / \mathrm{L}$ $+\mathrm{MI} / \mathrm{R}$ group; ${ }^{c c} \mathrm{P}<0.01$ compared with the TQ $2.5 \mu \mathrm{mol} / \mathrm{L}+\mathrm{MI} / \mathrm{R}$ group; ${ }^{\mathrm{P}}<0.05$ compared with the TQ $5 \mu \mathrm{mol} / \mathrm{L}+\mathrm{MI} / \mathrm{R}$ group; and dd $\mathrm{P}<0.01$ compared with the TQ 5 $\mu \mathrm{mol} / \mathrm{L}+\mathrm{MI} / \mathrm{R}$ group.

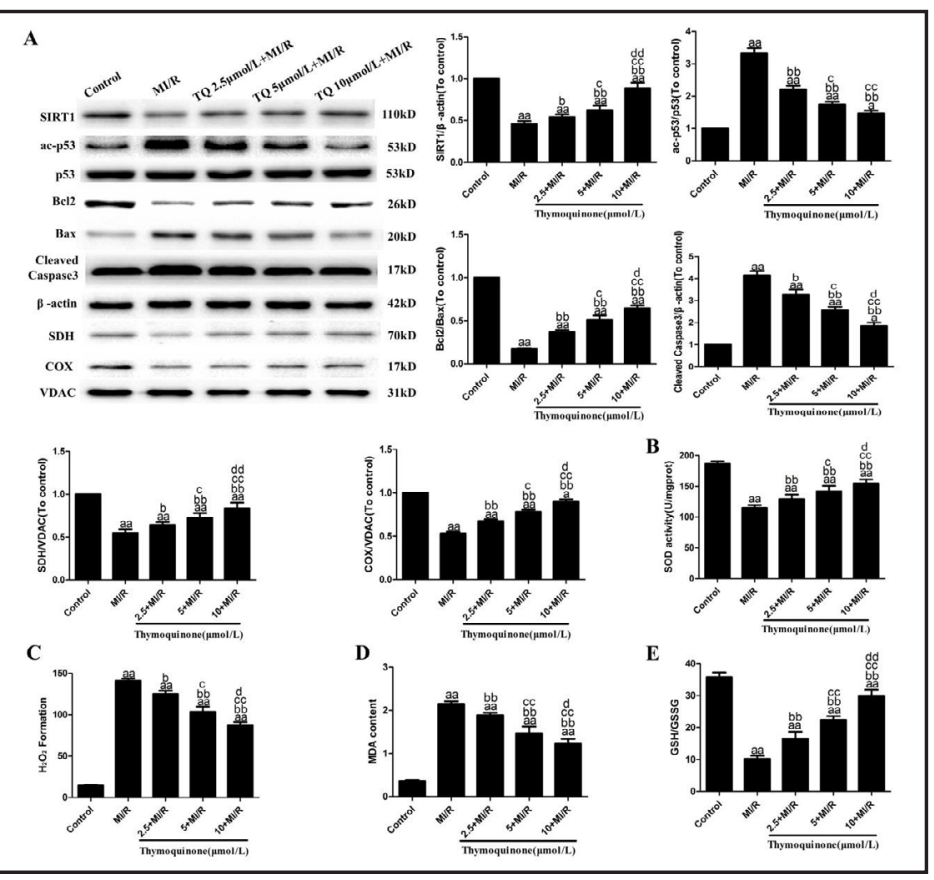


Fig. 4. Effects of $\mathrm{TQ}$ and sirtinol pretreatment on LDH release, cardiac function, and infarct size of MI/R-injured isolated rat hearts. (A) Representative diagrams of cardiac function. (B) LDH release. (C) Representative images of hearts. All results are expressed as the mean $\pm \mathrm{SD}, \mathrm{n}=6 .{ }^{\mathrm{a}} \mathrm{P}<0.05$ compared with the control group; ${ }^{a} \mathrm{P}<0.01$ compared with the control group; ${ }^{\mathrm{b}} \mathrm{P}<0.05$ compared with the MI/R group; ${ }^{b b} \mathrm{P}<0.01$ compared with the MI/R group; ${ }^{c} \mathrm{P}<0.05$ compared with the TQ 2.5

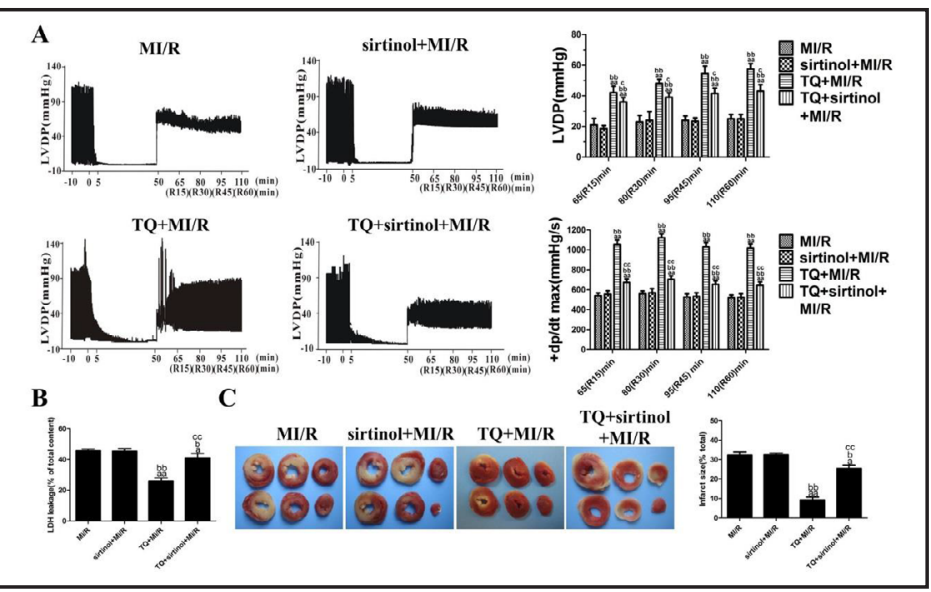
$\mu \mathrm{mol} / \mathrm{L}+\mathrm{MI} / \mathrm{R}$ group; and ${ }^{\mathrm{cc}} \mathrm{P}<0.01$ compared with the TQ $2.5 \mu \mathrm{mol} / \mathrm{L}+\mathrm{MI} / \mathrm{R}$ group. Sirtinol, the SIRT1 inhibitor; the hearts were treated with TQ or with an admixture of $3.75 \mu \mathrm{mol} / \mathrm{L}$ sirtinol 5 min prior to the MI/R experiment.

However, sirtinol pretreatment could increase the LDH release (compared with the TQ + MI/R group, $\mathrm{P}<0.01$ ). Heart images after TTC staining are shown in Fig. 4C. As could be seen, the percentage of infarct area was decreased to $9.29 \pm 1.48 \%$ after TQ pretreatment, which could be outstandingly increased to $25.44 \pm 1.73 \%$ by sirtinol treatment (compared with the $\mathrm{TQ}+\mathrm{MI} / \mathrm{R}$ group, $\mathrm{p}<0.01$ ). Additionally, the sirtinol $+\mathrm{MI} / \mathrm{R}$ treatment made no remarkable difference to infarct size (compared with the MI/R group, $p>0.05$ ).

Effects of $T Q$ and sirtinol pretreatment on mitochondrial oxidative damage of $M I / R$ injured isolated rat hearts

As illustrated in Fig. 5, TQ treatment promoted the activity of mitochondrial SDH, COX, and SOD (Fig. 5B, P < 0.01) but decreased the levels of mitochondrial MDA (Fig. 5D, $\mathrm{P}<0.01$ ) and $\mathrm{H}_{2} \mathrm{O}_{2}$ (Fig. $5 \mathrm{C}, \mathrm{P}<0.01$ ). Moreover, sirtinol treatment down-regulated the expression of SDH and COX, and decreased mitochondrial SOD activity in the TQ + sirtinol + MI/R group (compared with the TQ $+\mathrm{MI} / \mathrm{R}$ group, $\mathrm{P}<0.01$ ). Consistently, sirtinol treatment also markedly increased the levels of mitochondrial MDA and $\mathrm{H}_{2} \mathrm{O}_{2}$ in the TQ + sirtinol + MI/R group compared with the TQ + MI/R groups.

Effects of TQ and sirtinol pretreatment on the expression levels of SIRT1, ac-p53, Bcl2, Bax and Cleaved Caspase 3 of MI/R-injured isolated rat hearts

Compared with the MI/R group, sirtinol + MI/R treatment showed no obvious influence on the expression levels of related proteins (Fig. 5, P > 0.05). In addition, sirtinol treatment down-regulated SIRT1 expression but increased acetylation of p53. However, it notably decreased the $\mathrm{Bcl} 2 / \mathrm{Bax}$ ratio and increase Cleaved Caspase-3 expression (compared with the TQ + MI/R group, $\mathrm{P}<0.01$ ).

Effects of TQ treatment on the viability and apoptosis of SIR-injured cardiomyocytes

Primary neonatal rat cardiomyocytes were prepared as described above. Briefly, normal cells were treated with different concentrations of TQ. TQ treatment at the concentrations of $1 \mu \mathrm{mol} / \mathrm{L}, 2 \mu \mathrm{mol} / \mathrm{L}, 4 \mu \mathrm{mol} / \mathrm{L}$, and $8 \mu \mathrm{mol} / \mathrm{L}$ resulted in cell viability of SIR-injured cardiomyocytes of $1.279 \pm 0.044,1.280 \pm 0.036,1.312 \pm 0.045$, and $1.227 \pm 0.061$, respectively, which had no effect on that $(1.281 \pm 0.045)$ of normal cells (Fig. 6C, $P>0.05$ ). In comparison, TQ treatment at the concentrations of $16 \mu \mathrm{mol} / \mathrm{L}$ and $32 \mu \mathrm{mol} / \mathrm{L}$ notably decreased cell viability to $1.158 \pm 0.064$ and $0.851 \pm 0.064$, respectively $(\mathrm{P}<0.01)$. Afterwards, cells were divided into 5 groups, the control, SIR, TQ $1 \mu \mathrm{mol} / \mathrm{L}+\mathrm{SIR}$, TQ $2 \mu \mathrm{mol} / \mathrm{L}+\mathrm{SIR}$ and TQ $4 \mu \mathrm{mol} / \mathrm{L}+\mathrm{SIR}$ groups. Notably, TQ was administered $24 \mathrm{~h}$ before SIR treatment, as described above. The KARGER 
Fig. 5. Effects of $\mathrm{TQ}$ and sirtinol pretreatment on SIRT1 and acetylation of p53, apoptosis, and oxidative stress injury in MI/Rinjured isolated rat hearts. (A) Representative western blotting results were presented. (B) SOD activity. (C) $\mathrm{H}_{2} \mathrm{O}_{2}$ formation. (D) MDA content. All results are expressed as the mean $\pm \mathrm{SD}$, $\mathrm{n}=6$. ${ }^{\mathrm{a}} \mathrm{P}<0.05$ compared with the $\mathrm{MI} / \mathrm{R}$ group; aa $\mathrm{P}<0.01$ compared with the $\mathrm{MI} / \mathrm{R}$ group; ${ }^{\mathrm{b}} \mathrm{P}<0.05$ compared with the sirtinol + MI/R group; ${ }^{b} \mathrm{P}<0.01$ compared with the sirtinol + MI/R group; ${ }^{\mathrm{P}}<0.05$ compared with the $\mathrm{TQ}+\mathrm{MI} / \mathrm{R}$ group; and ${ }^{c c} \mathrm{P}<0.01$ compared with the TQ + MI/R group.

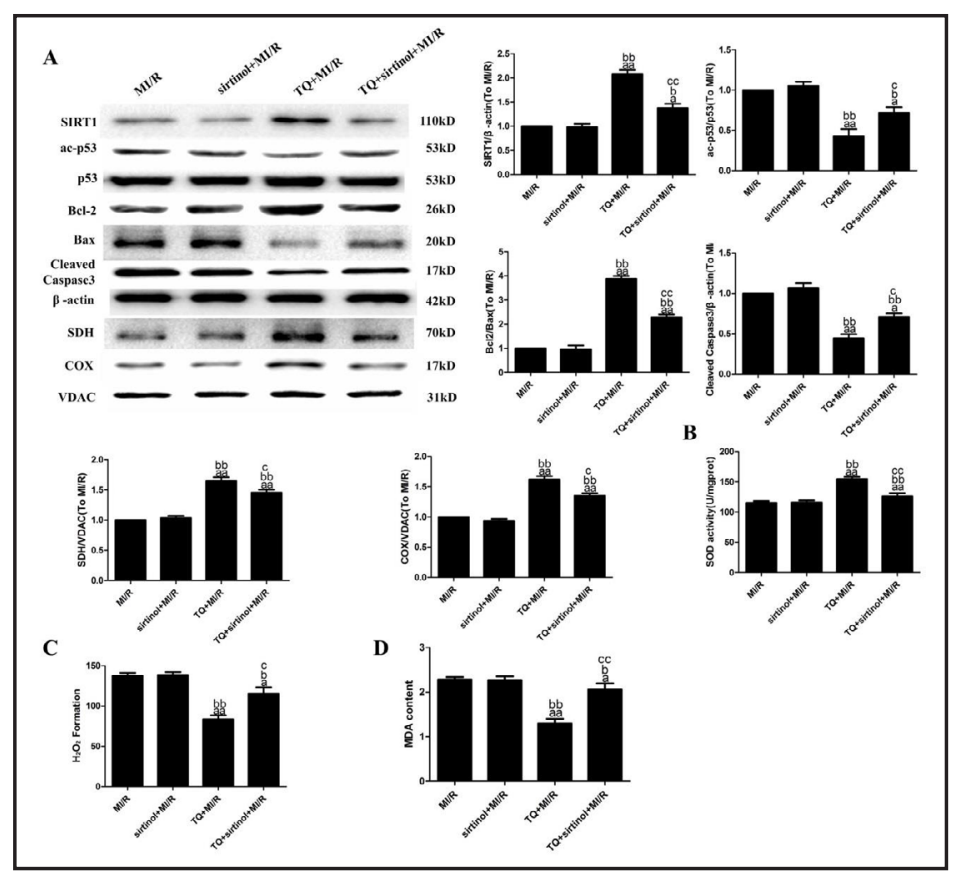

experiment was scheduled based on our previous results from previous studies. Compared with the control group with the cell viability of $1.037 \pm 0.072$, SIR treatment could obviously decrease the cell viability to $0.519 \pm 0.097$ (Fig. $6 \mathrm{~A}, \mathrm{P}<0.01$ ). Additionally, TQ treatment at the concentrations of $1 \mu \mathrm{mol} / \mathrm{L}, 2 \mu \mathrm{mol} / \mathrm{L}$ and $4 \mu \mathrm{mol} / \mathrm{L}$ could reverse cell viability to $0.637 \pm 0.061,0.741 \pm 0.035$ and $0.843 \pm 0.087$, respectively, among which, $4 \mu \mathrm{mol} / \mathrm{L} \mathrm{TQ}$ treatment appeared to be the most effective dose $(\mathrm{P}<0.01)$. In addition, results of TUNEL staining suggested that SIR treatment had resulted in a remarkably increased apoptotic ratio of $24.87 \pm 1.21 \%$ (Fig. $6 \mathrm{C}, \mathrm{P}<0.01$ ). In comparison, TQ treatment could reduce the apoptotic ratio in a concentrations-dependent manner, among which, $4 \mu \mathrm{mol} / \mathrm{L}$ TQ treatment could lower the apoptotic ratio to $12.22 \pm 0.74 \%$ (Fig. 6B, P < 0.01). Representative images of cell morphology are presented in Fig. 6.

Effects of TQ and SIRT1 SiRNA pretreatment on the viability and apoptosis of SIR-injured cardiomyocytes

Results in cardiomyocytes after TQ and SIRT1 siRNA pretreatment were consistent with those obtained from the isolated rats. In brief, TQ was administered $24 \mathrm{~h}$ prior to SIR treatment as described above. Compared with the SIR group, SIRT1 siRNA + SIR treatment made no obvious difference to viability or apoptosis ( $\mathrm{P}>0.05)$. Conversely, TQ pretreatment markedly enhanced cell viability following SIR. As observed microscopically, TQ pretreatment resulted in elevated cell contraction compared with that of the SIR group. Compared with the TQ + SIR group with cell viability of $0.889 \pm 0.078$ SIRT1 siRNA pretreatment almost abolished the protective effect of TQ pretreatment on cell viability, which had decreased to $0.687 \pm 0.058$ (Fig. 7A, $\mathrm{P}<0.01$ ). Furthermore, results of TUNEL staining demonstrated that SIRT1 siRNA treatment prominently increased the apoptotic ratio to $20.29 \pm 1.22 \%$ (compared with the TQ + SIR group, $14.13 \pm 1.31 \%$, Fig. 7B, P < 0.01). Representative images of cell morphology are presented in Fig. 7.

Effects of TQ and SIRT1 SiRNA pretreatment on mitochondrial oxidative damage of SIRinjured cardiomyocytes

As demonstrated in Fig. 8, compared with the SIR group, SIRT1 siRNA + SIR treatment exerted no marked influence on mitochondrial oxidative damage. Moreover, compared with the TQ + SIR group, SIRT1 siRNA treatment down-regulated expression of SDH and COX, 
Fig. 6. Effects of TQ pretreatment on viability and apoptosis of SIR-injured cardiomyocytes. (A) Representative images of cell morphology were presented. (B) Representative TUNEL staining images were presented. (C) Effects of TQ treatment on viability of normal cardiomyocytes. All results are expressed as the mean $\pm S D$, $\mathrm{n}=6$. ${ }^{\mathrm{a}} \mathrm{P}<0.05$ compared with the control group; aap $<0.01$ compared with the control group; ${ }^{b} \mathrm{P}<0.05$ compared with the SIR group; ${ }^{b b} \mathrm{P}<0.01$ compared with the SIR group; ${ }^{\mathrm{C}} \mathrm{P}<0.05$ compared with

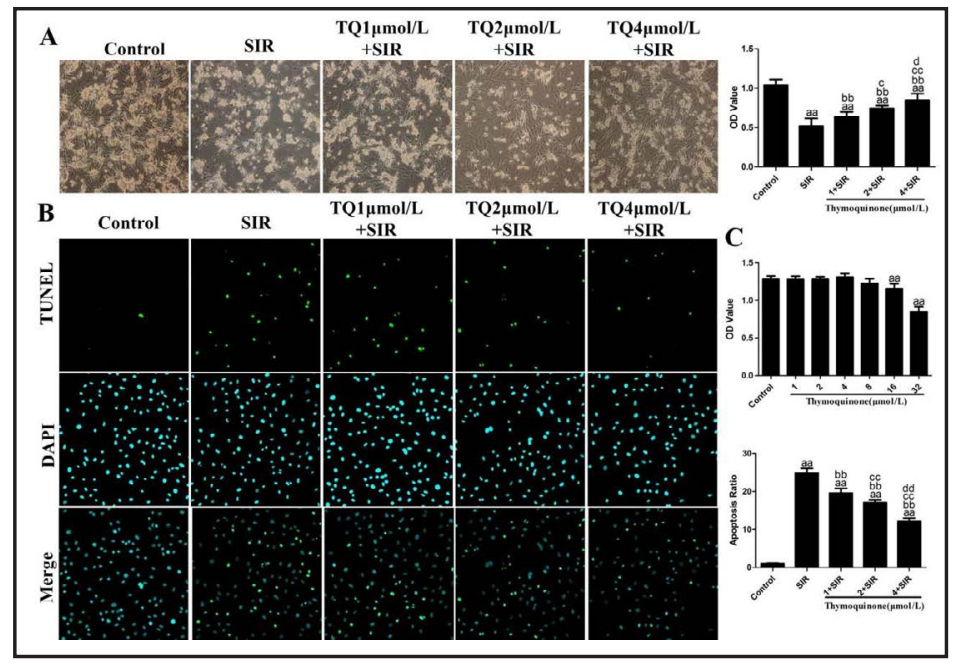
the TQ $2.5 \mu \mathrm{mol} / \mathrm{L}+$ SIR group; ${ }^{c c} \mathrm{P}<0.01$ compared with the TQ $2.5 \mu \mathrm{mol} / \mathrm{L}+\mathrm{SIR}$ group; ${ }^{\mathrm{d}} \mathrm{P}<0.05$ compared with the TQ $5 \mu \mathrm{mol} / \mathrm{L}+\mathrm{SIR}$ group; and ${ }^{\text {dd }} \mathrm{P}<0.01$ compared with the TQ $5 \mu \mathrm{mol} / \mathrm{L}+$ SIR group. SIR, simulated ischemia/reperfusion $(2$ $\mathrm{h} / 4 \mathrm{~h})$.

Fig. 7. Effects of TQ and SIRT1 siRNA pretreatment on the viability and apoptosis of SIRinjured cardiomyocytes.

Representative images of cell morphology. (B) Representative TUNEL staining images. All results are expressed as the mean $\pm \mathrm{SD}$, $\mathrm{n}=6$. ${ }^{\text {aa }} \mathrm{P}<0.01$ compared with the SIR group; ${ }^{\text {bb }} \mathrm{P}<0.01$ compared with the SIRT1 siRNA + SIR group; and ${ }^{c} \mathrm{P}<0.01$ compared with the TQ + SIR group.

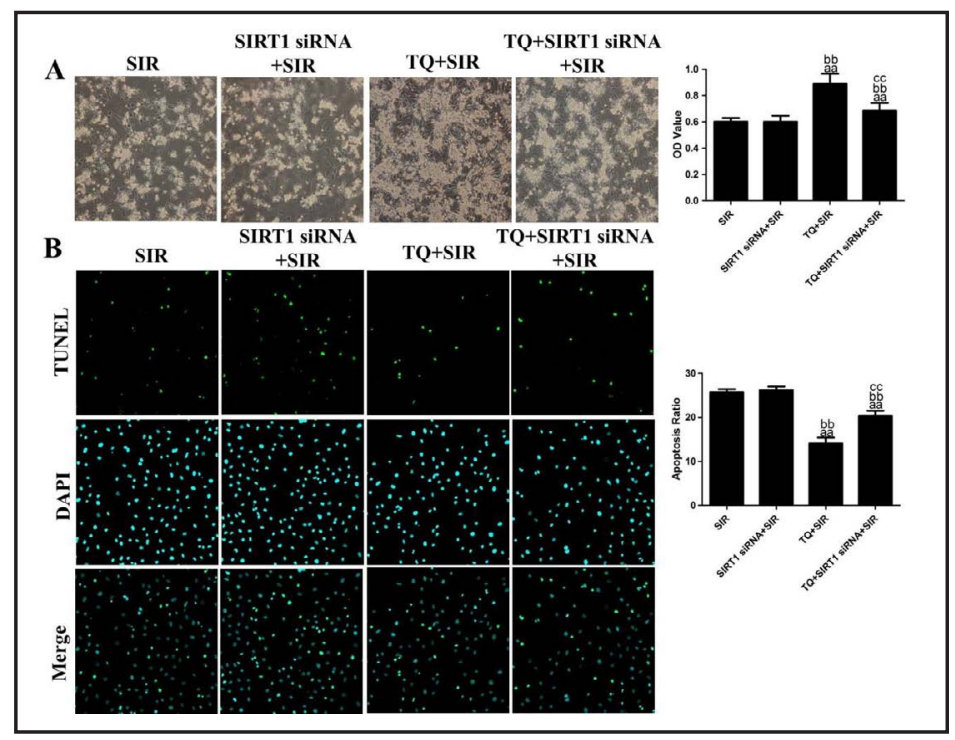

increased the levels of mitochondrial MDA (Fig. 8D, $\mathrm{P}<0.01$ ) and $\mathrm{H}_{2} \mathrm{O}_{2}$ (Fig. $8 \mathrm{C}, \mathrm{P}<0.01$ ), and decreased the activity of mitochondrial SOD (Fig. 8B, P < 0.01).

Effects of TQ and SIRT1 siRNA pretreatment on the expression levels of SIRT1, ac-p53, Bcl2, Bax, and Cleaved Caspase-3 of SIR-injured cardiomyocytes

As observed in Fig. 8, compared with the IR group, SIRT1 siRNA + SIR treatment made no marked difference to the expression levels of related proteins in SIR-injured cardiomyocytes $(\mathrm{P}>0.05)$. SIRT1 siRNA treatment down-regulated SIRT1 expression but enhanced acetylation of p53; there was also notable decrease in the Bcl2/Bax ratio and increased Cleaved Caspase-3 expression (compared with the TQ + SIR group, $\mathrm{P}<0.01$ ).

\section{Discussion}

Timely reperfusion has been verified to be the most efficient method to reduce myocardial infarct size. Undoubtedly, MI/R will aggravate the injury, which may account for up to $50 \%$ of the final infarct size. Unfortunately, no effective therapy for preventing $\mathrm{MI} / \mathrm{R}$ 
Fig. 8. Effects of TQ and SIRT1 siRNA pretreatment on SIRT1 and acetylation of p53, apoptosis and oxidative stress injury in SIR-injured cardiomyocytes. (A) Representative western blotting results were presented. (B) SOD activity. (C) $\mathrm{H}_{2} \mathrm{O}_{2}$ formation. (D) MDA content. All results are expressed as the mean $\pm S D, n=6$. ${ }^{\text {a }}<0.05$ compared with the SIR group; ${ }^{\mathrm{P}}<0.05$ compared with the SIR group; ${ }^{\text {aa }} \mathrm{P}<0.01$ compared with the SIR group; ${ }^{\mathrm{b}} \mathrm{P}<0.05$ compared with the SIRT1 siRNA + SIR group;

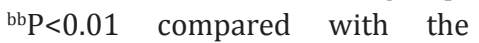
SIRT1 siRNA + SIR group; ${ }^{\mathrm{P}}<0.05$ compared with the TQ + SIR group; and ${ }^{c} \mathrm{P}<0.01$ compared with the TQ + SIR group.

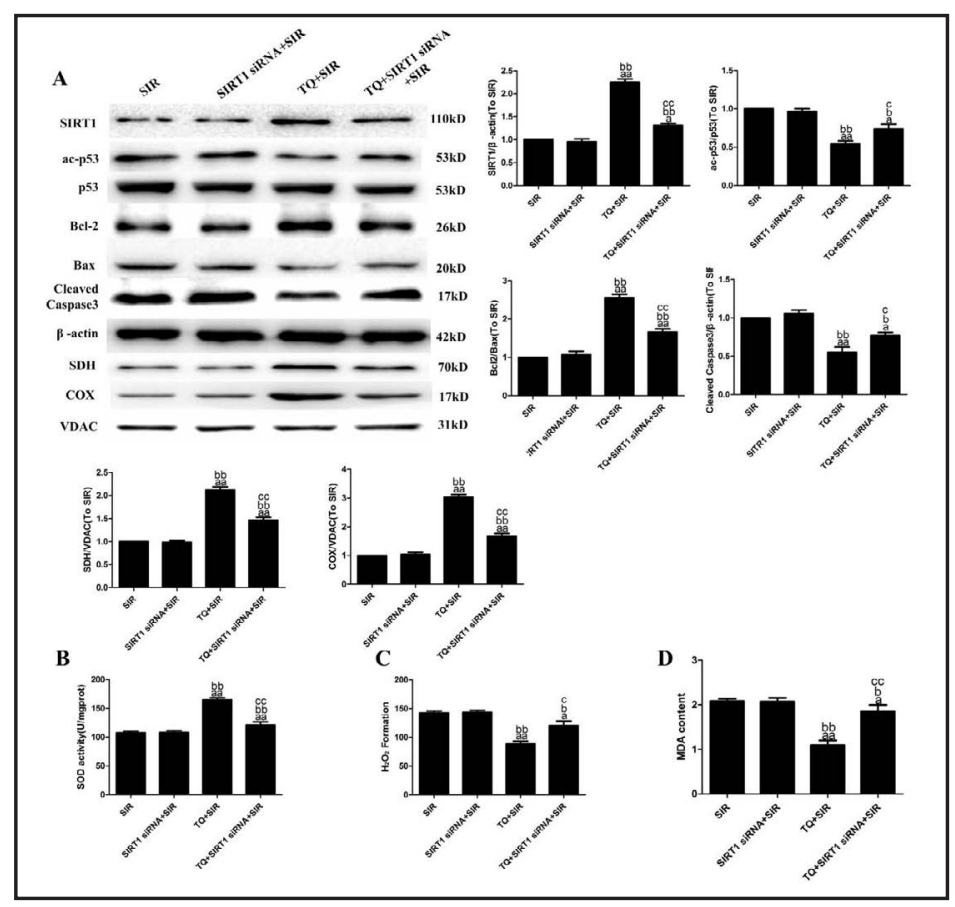

injury is available currently, despite the tremendous efforts that have been made [35].

In addition, mitochondrial permeability transition pore (mPTP), oxidative stress, intracellular calcium overload, and inflammation have been identified in previous studies to be the main mediators of MI/R injury. Specifically, the non-selective opening of MPTP at the beginning of reperfusion will result in mitochondrial oxidative stress, which may lead to mitochondrial matrix swelling, uncoupling of mitochondrial oxidative phosphorylation and apoptotic cell death. Mitochondrial oxidative stress is the major cause of mitochondrial dysfunction, which will in turn aggravate mitochondrial oxidative damage, ultimately exacerbating the MI/R injury. Therefore, protecting mitochondria from oxidative damage is a rational therapeutic approach to attenuate MI/R injury [35, 36].

SIRT 1 is an $\mathrm{NAD}^{+}$dependent histone deacetylase involved in many biological processes, including gene silencing, differentiation, cell survival, metabolism, and longevity. Moreover, SIRT 1 can also deacetylate and modulate the activities of various transcription factors and non-histone substrates, such as NF- $\mathrm{B}$, PGC1, FOXO, MyoD, and ac-p53 [27, 37]. Notably, SIRT 1 activation has a protective effect on the heart. For instance, Hsu and colleagues verified that SIRT 1 activation can protect the heart from IR injury by up-regulating antioxidants and down-regulating pro-apoptotic molecules, which was achieved through the activation of FOXO 1 and decreases in oxidative stress [27]. Ding et al. suggested that overexpression of SIRT 1 could attenuate diabetes-exacerbated MI/R injury and oxidative stress through activation of eNOS in diabetic rats [38]. p53, a tumor suppressor protein, plays a critical role in cell cycle control and apoptosis. Notably, induction of cell apoptosis is one of the most dramatic responses to p53 activation, which is achieved through the up-regulation of pro-apoptotic molecules $[39,40]$. Another study has also shown that deacetylation of p53 by overexpression of SIRT 1 exerts a protective effect on H9c2 cardiac myoblasts against hypoxia/reoxygenation injury [41]. Hence, targeting the SIRT1-p53 signaling pathway may serve as a potential therapy for MI/R injury. In the present study, the protective effects of TQ pre-conditioning on hearts or cardiomyocytes isolated from rats subjected to IR injury, as well as the correlation of such cardioprotective effect with the SIRT 1 signaling pathway were explored.

We found that TQ displays a cardioprotective effect, which can up-regulate SIRT 1 expression in the Langendorff IR model (Fig. 2). Interestingly, TQ can also up-regulate SIRT 1 
expression in normal isolated rat heart, indicating that TQ may be a SIRT 1 activator (Fig. 1E). Additionally, resveratrol, a SIRT 1 activator, is known to possess many beneficial biological activities. For instance, resveratrol can ameliorate doxorubicin-induced cardiotoxicity in aged hearts through restoring the SIRT1 activity, thus attenuating the USP7-related catabolic/ pro-apoptotic signaling [42]. Consequently, it can be easily speculated that TQ may exert its cardioprotective effect through activation of the SIRT 1 signaling pathway. Meanwhile, studies have indicated that SIRT 1 activation can attenuate the mitochondrial oxidative stress and maintain mitochondrial function. For instance, SIRT1 can alleviate high glucose-induced insulin resistance through ameliorating the mitochondrial dysfunction, which is achieved through the SIRT1-SIRT3-mitochondrial complex I pathway [43]. Curcumin pretreatmentinduced SIRT1 activation can attenuate mitochondrial oxidative damage resulting from MI/R injury [32]. In this study, we find that TQ can attenuate MI/R injury by reducing mitochondrial oxidative stress in isolated rat hearts or cardiomyocytes, which is achieved through activation of the SIRT 1 signaling. As shown in Fig. 3, TQ pretreatment can outstandingly up-regulate the expression of SIRT 1 and raise the GSH/GSSG ratio. Consistent with this protective effect, mitochondrial SOD activity was also elevated, whereas the production of $\mathrm{H}_{2} \mathrm{O}_{2}$ and MDA had obviously declined. Nevertheless, activity of mitochondrial SDH and COX was elevated. In summary, the mitochondrial oxidative damage caused by MI/R injury was remarkably attenuated and mitochondrial function also evidently improved.

This study showed that TQ preconditioning exerts a protective effect on neonatal rat cardiomyocytes, which is attained through activating the SIRT1 signaling pathway. A previous study has shown that deacetylation of p53 by up-regulating SIRT1 expression can protect cardiomyocytes from doxorubicin-induced cell apoptosis [44]. In the present study, TQ treatment can activate SIRT1 and suppress acetylation of p53. In contrast, Bcl2 expression is up-regulated, whereas expression of Bax and cleaved caspase- 3 is downregulated. All these findings indicate that TQ can reduce IR injury-induced cardiomyocyte apoptosis through the deacetylation of p53 by activating SIRT1.

However, the protective effects of $\mathrm{TQ}$ on the isolated rat hearts or neonatal rat cardiomyocytes subjected to IR injury can be abolished by sirtinol or SIRT1 siRNA treatment, demonstrating that TQ treatment may attenuate the MI/R injury by activating the SIRT1 signaling.

To the best of our knowledge, the present study is the first to elucidate the relationship between TQ treatment and SIRT1 signaling during MI/R injury. In summary, our findings suggest that TQ preconditioning exerts a profound and efficient cardioprotective effect against MI/R injury, which appears to be largely dependent on the activation of SIRT1 signaling by attenuating mitochondrial oxidative damage and reducing cardiomyocyte apoptosis. These results indicate that TQ may be a promising candidate for treating MI/R injury in cardiac surgery and ischemic heart disease. However, further studies are needed to clarify the precise mechanisms and to investigate the clinical applications of TQ.

\section{Acknowledgements}

This research was financially supported by the National Natural Science Foundation of China (NO. 81473132, 81603314, 81503285).

\section{Disclosure Statement}

The authors declare to have no competing interests.

\section{References}

1 Yellon DM, Hausenloy DJ: Myocardial reperfusion injury. N Engl J Med 2007;357:1121-1135.

2 Hausenloy DJ, Yellon DM: Myocardial ischemia-reperfusion injury: a neglected therapeutic target. J Clin Invest 2013;123:92-100. 


\section{Cellular Physiology Cell Physiol Biochem 2018;47:1193-1206 and Biochemistry Published online: June 18, $2018 \quad \begin{aligned} & \text { DOI: 10.1159/000490216 } 2018 \text { The Author(s). Published by S. Karger AG, Basel } \\ & \text { www.karger.com/cpb }\end{aligned}$}

Lu et al.: Thymoquinone Attenuates Myocardial Ischemia/Reperfusion Injury

-3 Xia Z, Li H, Irwin MG: Myocardial ischaemia reperfusion injury: the challenge of translating ischaemic and anaesthetic protection from animal models to humans. Br J Anaesth 2016;117:ii44-ii62.

-4 Zhang X, Liang X, Lin X, Zhang S, Huang Z, Chen C, Guo Y, Xuan F, Xu X, Huang R: Mechanism of the protective effect of Yulangsan flavonoid on myocardial ischemia/reperfusion injury in rats. Cell Physiol Biochem 2014;34:1050-1062.

5 Du L, Shen T, Liu B, Zhang Y, Zhao C, Jia N, Wang Q, He Q: Shock wave therapy promotes cardiomyocyte autophagy and survival during hypoxia. Cell Physiol Biochem 2017;42:673-684.

6 Pell VR, Chouchani ET, Frezza C, Murphy MP, Krieg T: Succinate metabolism: a new therapeutic target for myocardial reperfusion injury. Cardiovasc Res 2016;111:134-141.

-7 Gholamnezhad Z, Havakhah S, Boskabady MH: Preclinical and clinical effects of Nigella Sativa and its constituent, thymoquinone: a review. J Ethnopharmacol 2016;190:372-386.

-8 Gali-Muhtasib H, Roessner A, Schneider-Stock R: Thymoquinone: a promising anti-cancer drug from natural sources. Int J Biochem Cell Biol 2006;38:1249-1253.

-9 Schneider-Stock R, Fakhoury IH, Zaki AM, Elbaba CO, Gali-Muhtasib HU: Thymoquinone: fifty years of success in the battle against cancer models. Drug Discov Today 2014;19:18-30.

10 Woo CC, Kumar AP, Sethi G, Tan KH: Thymoquinone: potential cure for inflammatory disorders and cancer. Biochem Pharmacol 2012;83:443-451.

11 Rajput S, Kumar BN, Banik P, Parida S, Mandal M: Thymoquinone restores radiation-induced TGF- $\beta$ expression and abrogates EMT in chemoradiotherapy of breast cancer cells. J Cell Physiol 2015;230:620-629.

12 Wang D, Qiao J, Zhao X, Chen T, Guan D: Thymoquinone inhibits IL-1 $\beta$-induced inflammation in human osteoarthritis chondrocytes by suppressing NF- $\mathrm{KB}$ and MAPKs signaling pathway. Inflammation 2015;38:2235-2241.

13 Chen L, Li B, Chen B, Shao Y, Luo Q, Shi X, Chen Y: Thymoquinone alleviates the experimental diabetic peripheral neuropathy by modulation of inflammation. Sci Rep 2016;6:31656.

14 Ojha S, Azimullah S, Mohanraj R, Sharma C, Yasin J, Arya DS, Adem A: Thymoquinone protects against myocardial ischemic injury by mitigating oxidative stress and inflammation. Evid Based Complement Alternat Med 2015;2015:143629.

15 Salem ML: Immunomodulatory and therapeutic properties of the Nigella sativa L. seed. Int Immunopharmacol 2005;5:1749-1770.

16 Abd-Elbaset M, Arafa EA, El Sherbiny GA, Abdel-Bakky MS, Elgendy AN: Thymoquinone mitigate ischemia-reperfusion-induced liver injury in rats: a pivotal role of nitric oxide signaling pathway. Naunyn Schmiedebergs Arch Pharmacol 2017;390:69-76.

17 Gökce EC, Kahveci R, Gökce A, Cemil B, Aksoy N, Sargon MF, Kısa Ü, Erdoğan B, Güvenç Y, Alagöz F: Neuroprotective effects of thymoquinone against spinal cord ischemia-reperfusion injury by attenuation of inflammation, oxidative stress, and apoptosis. J Neurosurg Spine 2016;24:949-959.

-18 Gülşen İ, Ak H, Çölçimen N, Alp HH, Atalay T, Demir İ, Balaharoğlu R, Rağbetli MÇ: Neuroprotective effects of thymoquinone on the hippocampus in a rat model of traumatic brain injury. World Neurosurg 2016;86:243-249.

19 Hammad FT, Lubbad L: The effect of thymoquinone on the renal functions following ischemia-reperfusion injury in the rat. Int J Physiol Pathophysiol Pharmacol 2016;8:152-159.

20 Erboga M, Aktas C, Kurt O, Uygur R, Caglar V, Turan BC, Topcu B, Fidanol Erboga Z, Gurel A, Ozen OA: Protective effects of thymoquinone on experimental testicular ischaemia-reperfusion injury: an apoptotic, proliferative and biochemical study. Andrologia 2016;48:222-230.

-21 Gonca E, Kurt Ç: Cardioprotective effect of Thymoquinone: A constituent of Nigella sativa L., against myocardial ischemia/reperfusion injury and ventricular arrhythmias in anaesthetized rats. Pak J Pharm Sci 2015;28:1267-1273.

22 Heart EA, Palo M, Womack T, Smith PJ, Gray JP: The level of menadione redox-cycling in pancreatic $\beta$-cells is proportional to the glucose concentration: role of NADH and consequences for insulin secretion. Toxicol Appl Pharmacol 2012;258:216-225.

23 Gray JP, Z. BD, Yuan T, Seeram N, Rebar R, Follmer R, Heart EA: Thymoquinone, a bioactive component of Nigella sativa, normalizes insulin secretion from pancreatic $\beta$-cells under glucose overload via regulation of malonyl-CoA. Am J Physiol Endocrinol Metab 2016;310:E394-404.

24 Gonfloni S, Iannizzotto V, Maiani E, Bellusci G, Ciccone S, Diederich M: P53 and Sirt1: Routes of metabolism and genome stability. Biochem Pharmacol 2014;92:149-156. 


\section{Cellular Physiology Cell Physiol Biochem 2018;47:1193-1206 \begin{tabular}{l|l} 
and Biochemistry Published online: June 18, 2018 & $\begin{array}{l}\text { (c) } 2018 \text { The Author(s). Published by S. Karger AG, Basel } \\
\text { www.karger.com/cpb }\end{array}$
\end{tabular}}

Lu et al.: Thymoquinone Attenuates Myocardial Ischemia/Reperfusion Injury

25 Zhang ZY, Hong D, Nam SH, Kim JM, Paik YH, Joh JW, Kwon CH, Park JB, Choi GS, Jang KY, Park CK, Kim SJ: SIRT1 regulates oncogenesis via a mutant p53 -dependent pathway in hepatocellular carcinoma. J Hepatol 2015;62:121-130.

26 Karandrea S, Yin H, Liang X, Slitt AL, Heart EA: Thymoquinone ameliorates diabetic phenotype in DietInduced Obesity mice via activation of SIRT-1-dependent pathways. Plos One 2017;12:e0185374.

27 Hsu CP, Zhai P, Yamamoto T, Maejima Y, Matsushima S, Hariharan N, Shao D, Takagi H, Oka S, Sadoshima J: Silent information regulator 1 protects the heart from ischemia/reperfusion. Circulation 2010;122:2170-2182.

28 Lau AW, Liu P, Inuzuka H, Gao D: SIRT1 phosphorylation by AMP-activated protein kinase regulates p53 acetylation. Am J Cancer Res 2014;4:245-255.

-29 Yang Y, Duan W, Jin Z, Yi W, Yan J, Zhang S, Wang N, Liang Z, Li Y, Chen W, Yi D, Yu S: JAK2/STAT3 activation by melatonin attenuates the mitochondrial oxidative damage induced by myocardial ischemia/reperfusion injury. J Pineal Res 2013;55:275-286.

30 Kiscsatári L, Varga Z, Schally AV, Gáspár R, Nagy CT, Giricz Z, Ferdinandy P, Fábián G, Kahán Z, Görbe A: Protection of neonatal rat cardiac myocytes against radiation-induced damage with agonists of growth hormone-releasing hormone. Pharmacol Res 2016;111:859-866.

31 Yang Y, Wang J, Yue L, Fan C, Shuai J, Lei Z, Di S, Xin Z, Wang B, Wu G, Li X, Li Z, Gao X, Dong Y, Qu Y: HO-1 signaling activation by pterostilbene treatment attenuates mitochondrial oxidative damage induced by cerebral ischemia reperfusion injury. Mol Neurobiol 2016;53:2339-2353.

-32 Yang Y, Duan W, Lin Y, Yi W, Liang Z, Yan J, Wang N, Deng C, Zhang S, Li Y, Chen W, Yu S, Yi D, Jin Z: SIRT1 activation by curcumin pretreatment attenuates mitochondrial oxidative damage induced by myocardial ischemia reperfusion injury. Free Radic Biol Med 2013;65:667-679.

-33 Fan C, Pan Y, Yang Y, Di S, Jiang S, Ma Z, Li T, Zhang Z, Li W, Li X, Reiter RJ, Yan X: HDAC1 inhibition by melatonin leads to suppression of lung adenocarcinoma cells via induction of oxidative stress and activation of apoptotic pathways. J Pineal Res 2015;59:321-333.

-34 Yang Y, Fan C, Deng C, Zhao L, Hu W, Di S, Ma Z, Zhang Y, Qin Z, Jin Z, Yan X, Jiang S, Sun Y, Yi W: Melatonin reverses flow shear stress-induced injury in bone marrow mesenchymal stem cells via activation of AMPactivated protein kinase signaling. J Pineal Res 2015;60:228-241.

35 Zhang P, Lu Y, Yu D, Zhang D, Hu W: TRAP1 provides protection against myocardial ischemia-reperfusion injury by ameliorating mitochondrial dysfunction. Cell Physiol Biochem 2015;36:2072-2082.

-36 Li T, Zhang P, Liu J, Zhou R, Li Q, You Z, Dian K: Protective effects of hemoglobin-based oxygen carrier given to isolated heart during ischemia via attenuation of mitochondrial oxidative damage. Free Radic Biol Med 2010;48:1079-1089.

37 Hori YS, Kuno A, Hosoda R, Horio Y: Regulation of FOXOs and p53 by SIRT1 modulators under oxidative stress. Plos One 2013;8:e73875.

-38 Ding M, Lei J, Han H, Li W, Qu Y, Fu E, Fu F, Wang X: SIRT1 protects against myocardial ischemia-reperfusion injury via activating eNOS in diabetic rats. Cardiovasc Diabetol 2015;14:143.

39 Wang D, Li Z, Zhang Y, Wang G, Wei M, Hu Y, Ma S, Jiang Y, Che N, Wang X: Targeting of microRNA-199a5 p protects against pilocarpine-induced status epilepticus and seizure damage via SIRT1-p53 cascade. Epilepsia 2016;57:706-716.

40 Amaral JD, Castro RE, Steer CJ, Rodrigues CM: p53 and the regulation of hepatocyte apoptosis: implications for disease pathogenesis. Trends Mol Med 2009;15:531-541.

41 Liu L, Wang P, Liu X, He D, Liang C, Yu Y: Exogenous NAD+ supplementation protects H9c2 cardiac myoblasts against hypoxia/reoxygenation injury via Sirt1-p53 pathway. Fundam Clin Pharmacol 2014;28:180-189.

-42 Sin TK, Tam BT, Yung BY, Yip SP, Chan LW, Wong CS, Ying M, Rudd JA, Siu PM: Resveratrol protects against doxorubicin-induced cardiotoxicity in aged hearts through the SIRT1-USP7 axis. J Physiol 2015;593:1887-1899.

43 Zhang HH, Ma XJ, Wu LN, Zhao YY, Zhang PY, Zhang YH, Shao MW, Liu F, Li F, Qin GJ: SIRT1 attenuates high glucose-induced insulin resistance via reducing mitochondrial dysfunction in skeletal muscle cells. Exp Biol Med 2015;240:557-565.

44 Zhang C, Feng Y, Qu S, Wei X, Zhu H, Luo Q Liu M, Chen G, Xiao X: Resveratrol attenuates doxorubicininduced cardiomyocyte apoptosis in mice through SIRT1-mediated deacetylation of p53. Cardiovasc Res 2011;90:538-545. 